\title{
The quest toward the Holy Grail of mechanical circulatory support
}

\author{
Francis D. Pagani, MD, PhD
}

\footnotetext{
From the Department of Cardiac Surgery, Cardiovascular Center, University of Michigan Health Systems, Ann Arbor, Mich.

Disclosures: Author has nothing to disclose with regard to commercial support.

Received for publication June 24, 2015; accepted for publication June 25, 2015; available ahead of print July 31, 2015.

Address for reprints: Francis D. Pagani, MD, PhD, Department of Cardiac Surgery, Cardiovascular Center, University of Michigan Health Systems, 1500 East Medical Center Drive, Ann Arbor, MI 48109 (E-mail: fpagani@ umich.edu).

J Thorac Cardiovasc Surg 2015;150:694-5

$0022-5223 / \$ 36.00$

Copyright $(2) 2015$ by The American Association for Thoracic Surgery

http://dx.doi.org/10.1016/j.jtcvs.2015.06.072
}

The modern quest to develop a clinically viable mechanical replacement of the failing human heart has challenged scientists and clinicians for more than 50 years. The continuing pursuit of a practical mechanical solution to the failing heart has been fostered by the inability of biological alternatives, including cardiac transplantation and gene or stem cell therapy, to adequately address both the magnitude and the severity of the problem of advanced heart failure. In 1957, Akutsu and Kolff reported the first experimental animal implant of a total artificial heart (TAH) that stimulated enormous interest in the field. ${ }^{1}$ However, the task of mechanically replicating the rhythmic efficiency and complexity of the human heart has brought continuing challenges and failures. Early TAH designs created pulsatile output from volume-displacement pumps, using unidirectional inlet and outlet valves in each chamber. These designs required tethering to an external pneumatic drive system, making permanent heart replacement largely impractical. Pulsatile designs with self-contained actuation mechanisms and transcutaneous power delivery systems were subsequently developed, some progressing to clinical investigation (eg, AbioCor TAH; Abiomed Inc, Danvers, Mass). ${ }^{2}$ However, issues with biocompatibility, large size, infection, and poor durability have continued to prevent clinical realization of the dream to permanently replace the human heart. To date, the only TAH that has been implanted with any important clinical impact, the SynCardia TAH-t (SynCardia Systems Inc, Tucson, Ariz), uses older technology (the Jarvik 7 TAH [Jarvik Heart Inc, New York, NY] was first implanted in humans in 1983) ${ }^{3}$ including volume displacement pumps and an external pneumatic actuation mechanism via transcutaneous air hoses. An important design update to this device has been the miniaturization of the drive system permitting greater patient mobility and discharge to home. However, this device is used almost exclusively for a bridge to transplant indication, although ongoing clinical success has again raised consideration of its use for permanent implantation.

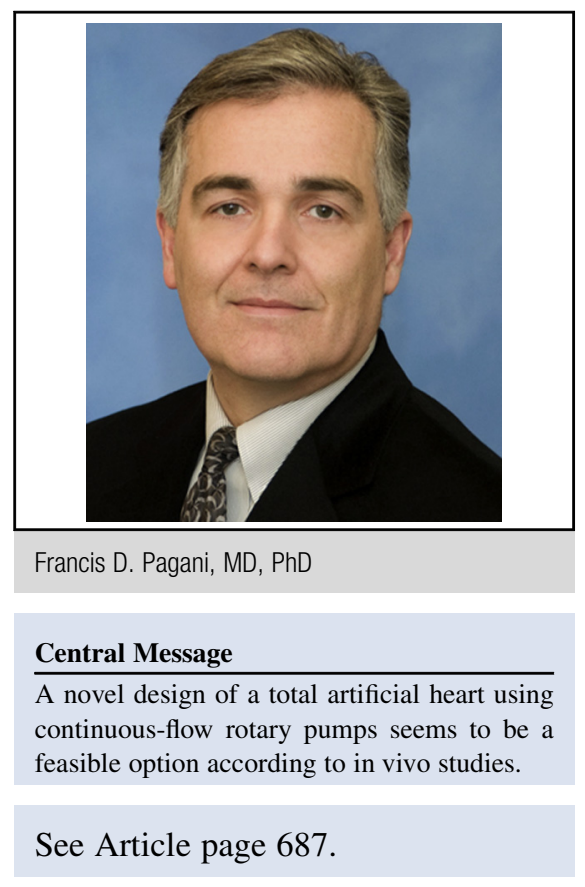

The ability of ventricular assist device (VAD) therapy to successfully support most patients with advanced heart failure without the need for total heart replacement has questioned the need for further research to develop a TAH solution. However, clinical scenarios such as severe biventricular failure, cardiac allograft failure, cardiac malignancies, untreatable cardiac dysrhythmias, and restrictive or obstructive cardiomyopathies not amenable to VAD therapy occur with sufficient frequency to justify ongoing TAH development and use. The success of applying continuous flow $(\mathrm{CF})$ rotary pump technology to VAD designs has stimulated a number of novel designs for TAH technology.

In this issue of the Journal, Karimov and colleagues ${ }^{4}$ at the Cleveland Clinic summarize data evaluating the in vivo pump performance of a unique TAH design using CF rotary pump technology. This design features a pump with a cylindrical rotating element supported by a fluidfilm hydrodynamic bearing. The length of the cylinder is approximately twice its diameter, and the cylinder is capped at both ends by centrifugal flow impellers. When the systemic-pulmonary circulation becomes imbalanced, the hypofunctioning side of the TAH is exposed to progressively increasing atrial pressure, which creates a hydrodynamic force on the spinning element and shifts the axis of rotation toward the side with lower atrial pressure. Each impeller is positioned in such a way with respect to the volute in which it is spinning that alignment is improved or worsened 
depending on whether the rotor shifts to the left or right. This change adjusts the relative outputs of the left and right sides of the device and continuously balances pumping efficiency without the need for sensors or feedback algorithms. The authors describe the use of this novel device in 17 animals, with 3 of the most recent animals surviving for the planned duration of the experiment $(30,90$, and 90 days, respectively). In vivo experiments demonstrated good hemodynamic performance without mechanical failure of the device. The majority of experimental failures were attributed to perioperative procedural-related issues, but the authors did describe important issues with potential design problems, including hemolysis due to thrombus depositions on the journal bearing encountered in 2 experiments and electrical failure of a controller chip causing pump stoppage in 1 case. A shorter motor design permitting a $20 \%$ reduction in the overall bearing length resolved the issue of hemolysis in the last 6 experimental studies. Another important finding at necropsy in the last 2 animals was the presence of an embolus caught on the right pump impellor, suggesting that venous thromboemboli could be an important problem for $\mathrm{CF}$ pumps designed with small tolerances.

Current and future designs of the TAH need to provide a small, self-contained drive unit that is physiologically responsive, balances right and left outputs, requires low power consumption, and has enhanced biocompatibility and a minimum 5-year durability. Along these lines, other notable efforts with TAH development are ongoing, including the CARMAT TAH (Velizy Villacoublay, France), ReinHeart TAH (Aachen, Germany), and BiVACOR TAH (Houston, Tex). ${ }^{5,6}$ The effort by Karimov and colleagues ${ }^{4}$ at Cleveland Clinic represents an important step in leveraging CF rotary pump technology toward TAH development and demonstrates the complexities of subtle design changes on performance. Whether CF rotary pump technology can be adapted to TAH designs to deliver a practical permanent replacement of the human heart with important clinical success is unknown, but this work represents an important step forward in the field.

\section{References}

1. Akutsu T, Kolff WJ. Permanent substitutes for valves and hearts. ASAIO J. 1958;4 230-4.

2. Dowling RD, Gray LA Jr, Etoch SW, Laks H, Marelli D, Samuels L, et al. Initial experience with the AbioCor implantable replacement heart system. J Thorac Cardiovasc Surg. 2004;127:131-41.

3. DeVries WC, Anderson JL, Joyce LD, Anderson FL, Hammond EH, Jarvik RK, et al. Clinical use of the total artificial heart. $N$ Engl J Med. 1984;310:273-8.

4. Karimov JH, Moazami N, Kobayashi M, Sale S, Such K, Byram N, et al. First report of 90-day support of two calves with a continuous flow total artificial heart. J Thorac Cardiovasc Surg. 2015;150:687-93.e1.

5. Cohn WE, Timms DL, Frazier OH. Total artificial hearts: past, present, and future Nat Rev Cardiol. June 2, 2015 [Epub ahead of print].

6. Gerosa G, Scuri S, Iop L, Torregrossa G. Present and future perspectives on total artificial hearts. Ann Cardiothorac Surg. 2014;3:595-602. 